\title{
Solución informática para el sector hotelero
}

\section{Information-technology solution for the hotel sector}

1 Jansel A. López Bouza Universidad de las Ciencias Informáticas, Habana, Cuba janselalb@estudiantes.uci.cu

2 Yordany Ponce Alderete

Universidad de las Ciencias Informáticas, Habana, Cuba yordanypa@estudiantes.uci.cu

3 Alejandro Sánchez Fernández Universidad de las Ciencias Informáticas, Habana, Cuba alejandrosf@estudiantes.uci.cu

4 Efraín Velastegui López https://orcid.org/0000-0002-7353-5853 Universidad Técnica de Babahoyo, Babahoyo, Ecuador evelasteguil@utb.edu.ec

Artículo de Investigación Científica y Tecnológica

Enviado: 25/12/2021

Revisado: $30 / 12 / 2021$

Aceptado: 15/01/2022

Publicado:26/02/2022

DOI: $\quad$ https://doi.org/10.33262/ap.v4i1.188

Cítese:

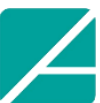

\section{Ciencia \\ Digital}

\section{c) $\$$}

López Bouza, J. A., Ponce Alderete, Y., Sánchez Fernández, A., \& Velastegui López, E. (2022). Solución informática para el sector hotelero. AlfaPublicaciones, 4(1), 197205. https://doi.org/10.33262/ap.v4i1.188

ALFA PUBLICACIONES, es una Revista Multidisciplinar, Trimestral, que se publicará en soporte electrónico tiene como misión contribuir a la formación de profesionales competentes con visión humanística y crítica que sean capaces de exponer sus resultados investigativos y científicos en la misma medida que se promueva mediante su intervención cambios positivos en la sociedad. https://alfapublicaciones.com

La revista es editada por la Editorial Ciencia Digital (Editorial de prestigio registrada en la Cámara Ecuatoriana de Libro con No de Afiliación 663) www.celibro.org.ec

Esta revista está protegida bajo una licencia Creative Commons AttributionNonCommercialNoDerivatives 4.0 International. Copia de la licencia: http://creativecommons.org/licenses/by-nc-nd/4.0/ 
Palabras claves: turismo, situación económica, solución informática, hotel.

\section{Keywords:}

tourism, economic situation, computer solution, hotel.

\section{Resumen}

El Turismo en Cuba ha representado durante muchos años un fuerte contribuyente económico al estado, reconociendo a esta área como fuente excepcional de ingreso, sobre todo para países en desarrollo, por tal motivo se hace imprescindible elevar la calidad del servicio en sus diferentes instituciones. Al ser las hoteleras una de las principales entidades turísticas se decidió hacer un software diseñado para las mismas. En vista a la situación económica por la que atraviesa el país y a la pandemia de la COVID-19 (pero no limitándose al tiempo de estadía de esta), se comenzó el desarrollo de una solución informática. Así fue como nació la idea de realizar una aplicación que gestione los servicios en el sector del turismo y permita mantener hasta cierto punto el distanciamiento entre los clientes y los trabajadores en un hotel; si, fue realizada desde un primer momento con vistas al trabajo realizado en hoteles, para mejorar y facilitar a su vez, el trabajo de este y la estancia del cliente, elevando la calidad y eficiencia de los servicios. El objetivo en sí del trabajo se enfoca en exponer las fases por las que ha transcurrido dicha aplicación y brindar una herramienta informática asequible, con la que solucionar dos problemas fundamentales que se están viviendo en el país hoy en día, y, aportar como estudiantes una ayuda en nuestro campo, que es la informática, a mejorar como país.

\section{Abstract}

Tourism in Cuba has represented a strong contributor for many years economic to the state, recognizing this area as an exceptional source of income, especially for developing countries, for this reason it is essential to raise the quality of service in its different institutions. As hotel companies are one of the main tourist entities, it was decided to make a software designed for themselves. In view of the economic situation the country is going through and the COVID-19 pandemic (but not limited to the length of stay of the same), the development of a computer solution began. This is how the idea of creating an application that manages services in the tourism sector and allows in a hotel; Yes, it was done from the beginning with a view to the work of the same and the stay of the customer, raising the quality and efficiency of services. The goal itself of the job focuses on exposing the phases through which said application has passed and providing an affordable computing tool, with which to solve two problems fundamentals that are being lived in 
the country today, and contribute as students some help in our field, which is computer science, to improve how country.

\section{Introducción}

Este trabajo contiene un valor práctico ya que a través de la actividad científica se espera encontrar alternativas posibles de aplicar en la práctica social y de esta manera poder satisfacer necesidades y expectativas.

La necesidad que se intenta satisfacer es la de facilitar y gestionar el trabajo en hoteles en lo relacionado a los servicios e información dentro del mismo (Mintur, 2021), ya que se ha dado el caso que los clientes no acuden a una actividad o no van a un área por desconocimiento de estas, al igual que les brinda la posibilidad a los usuarios de pedir servicios o productos desde su habitación con su teléfono celular o laptop, desde una página web (aunque se trabaja en el desarrollo de la aplicación móvil) (Colombia.Travel, 2021). Para esta aplicación desde un principio se usó de objeto de estudio a personas que se habían hospedado en algún hotel de Cuba, se les realizó una entrevista breve y se concluyó que en algunos de los casos a estos no se les daba alguna información importante de una actividad a realizarse o no habían visitado un área del hotel por desconocer su existencia (Eumed, 2021). Dado los datos iniciales se puso entonces manos a la obra y fue donde un equipo de tres estudiantes decidió proveer de una solución informática para los problemas dados en el estudio inicial realizado.

Otro elemento novedoso de dicha aplicación es la incorporación de diferentes sectores de la población al mundo hotelero, debido a que cualquier persona u organismo que posea esta y se registre en la misma ya estaría disponible su hotel en la página web para el deleite de los futuros clientes (Geroldi, 2019).

Se espera contribuir a la informatización en Cuba en esta área y a mejorar estadía de los clientes en un determinado hotel. Se mantiene a lo largo del trabajo el objetivo de explicar y describir las fases que tuvieron lugar en el proceso de desarrollo del software.

\section{Metodología}

La investigación se realizó en la modalidad bibliográfica o documentada. Bibliográfica porque esta dio lugar a que se ampliaran y profundizaran diferentes enfoques, teorías, conceptos y criterios de distintos autores acerca del tema en cuestión. También sostiene un enfoque experimental ya que se intenta a raíz de esta buscar la mejor solución que se adapte a las necesidades y a los requisitos que se exponen en este trabajo. 


\section{Herramientas e instrumentos de desarrollo}

La investigación se realizó en la modalidad bibliográfica o documentada. Bibliográfica porque esta dio lugar a que se ampliaran y profundizaran diferentes enfoques, teorías, conceptos y criterios de distintos autores acerca del tema en cuestión (Xpertosolutions, 2021).

- Kotlin: Además de clases y métodos clásicos de la programación orientada a objetos, soporta también programación por procedimientos y el uso de funciones. Soporta a su vez la Inter polarización de variables dentro de cadenas de texto lo que resultó bastante útil para realizar las sentencias MySQL. También se puede evidenciar que es un lenguaje moderno, lo que refleja en su sintaxis, es plenamente interoperable con código Java y fue creado por JetBrains en sus oficinas de San Petersburgo (Rusia).

- Bootstrap: Un marco de trabajo para desarrollo web responsive con HTML (Lenguaje de marcado de hipertexto por sus siglas en inglés), CSS (hojas de estilo en cascada por sus siglas en inglés) y JavaScript, además de la biblioteca jQuery para facilitarnos la interactividad con el usuario.

- Django: Uno de los más populares frameworks, este está programado en Python, respeta el patrón de diseño conocido como Modelo-vista-controlador, ha sido usado en grandes aplicaciones web como Instagram, National Geographic, Pinterest, etc. (Django, 2021).

\section{Figura 1}

\section{Herramientas utilizadas}

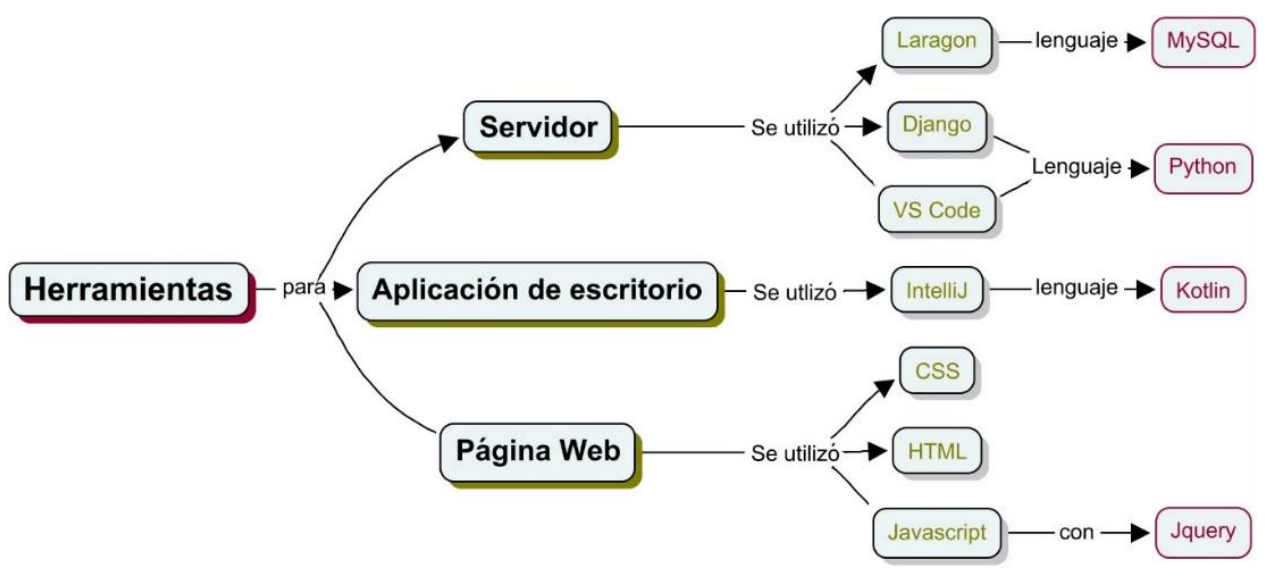




\section{Resultados}

\section{El software}

Al abrir el software se encuentra siempre una ventana de login, donde puede iniciar sesión con su usuario y contraseña o registrarse si es primera vez que lo abre y no se encuentra en la base de datos. A la hora del registro aparecen unos sencillos pasos. En el primer paso contiene campos a rellenar como son usuario, contraseña, repetir contraseña, nombre del hotel, teléfono, correo, provincia y municipio. Todos los campos son obligatorios, excepto en el caso de teléfono y correo que se puede avanzar teniendo solo uno de los anteriores. El segundo paso consiste en agregar un texto de descripción para el hotel y por último solo queda agregar fotos, la cantidad que el usuario desee, luego se presiona continuar y ya queda agregado el hotel, se puede ahora iniciar sesión y contar con los servicios del software.

Lo primero que se genera al iniciar sesión y entrar en el software es el menú, en este aparecen como prioridad los pedidos de los clientes registrados a actividades a realizar en el hotel. En la barra superior se encuentra la sección "registro", en ella aparecen las opciones de tienda, restaurante, cliente, habitación, área, producto y actividad. Al presionar en cualquiera de estas opciones aparecerá otra ventana con las opciones de agregar, actualizar, eliminar, ver y en algunos casos agregar imagen. La otra sección del menú en la barra superior se nombra "Hotel", en esta solo se pueden realizar cambios a los datos ya introducidos en el hotel a la hora de realizar el registro. Por último, se encuentra la sección "contactar" para brindar soporte, reportar sobre fallos (ya que ningún sistema está libre de fallos) y brindar sugerencias, para esto se encuentra una ventana con las fotos, correos (UCI y Gmail) y nombre completo de los desarrolladores.

\section{Figura 2}

Ventana de login en la aplicación de escritorio

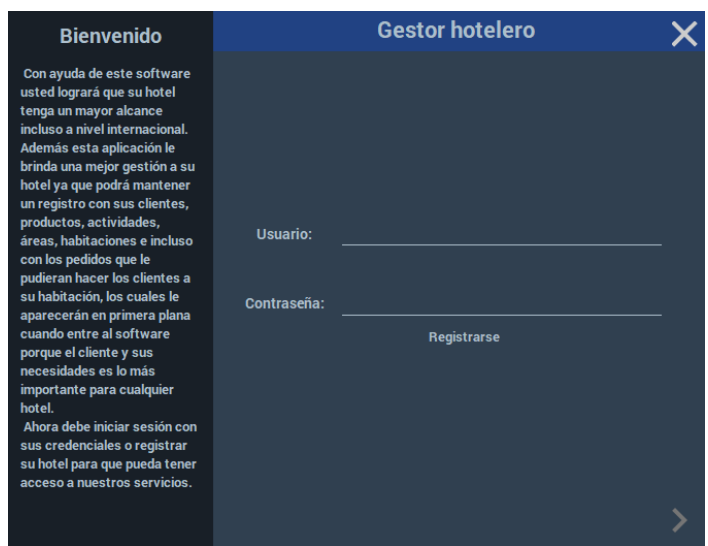




\section{$\underline{\text { El sitio }}$}

En la portada de la página se muestran imágenes relacionadas con el entorno natural, a la vez de un inicio de sesión y la posibilidad de registrarse en el sitio.

En el sitio se ven reflejadas variedades de actividades recreativas, se brinda amplia posibilidad de interactuar con ellas, se muestran los terrenos cercanos de nuestra casa de alojamiento y las atenciones ofrecidas que pueden solicitar los clientes. El buffet es otra de las cualidades presentes en nuestro sitio, con diversidad de alimentos, algo fascinante y de seguro muy bien agradecido por los huéspedes. Además, a lo largo del viaje por la aplicación web, se mantiene descripciones y frases de todos los elementos representados en la misma dadas por la misma institución o aportadas por el equipo de desarrollo para el cliente.

Por último, se observan los recorridos de bienvenida que ofrece la casa de hospedaje en caso de tenerlos, los paseos virtuales para familiarizar a los futuros clientes con el lugar donde se hospedarán, aquí se encuentra: observar las áreas, el menú de los restaurantes, los productos con los que cuenta en el momento, etcétera.

\section{Figura 3}

Áreas de un hotel en la página web
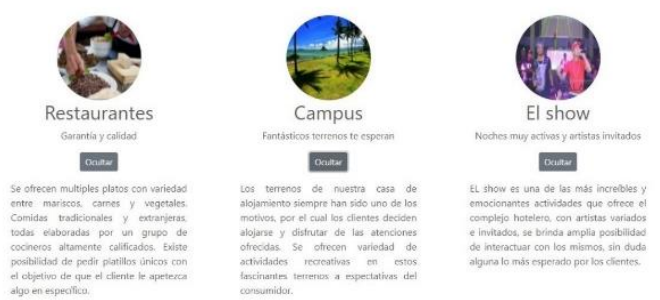

\section{Figura 4}

Un área en detalles en la página web

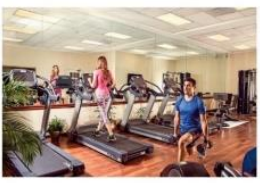




\section{El servidor}

El proyecto en Django fue nombrado "hotel" y contiene dos aplicaciones "api" esta aplicación se encarga de gestionar la base de datos, a través de las vistas de rest framework la cual le ofrece el acceso al cliente a ciertos datos en dependencia de su nivel de autorización, estos datos se transmiten como "Json"; la otra aplicación es "ui" la cual se encarga de renderizar el contenido gráfico que verá el cliente y ofrecerle el acceso a este.

\section{Figura 5}

Archivo models.py (Backend)

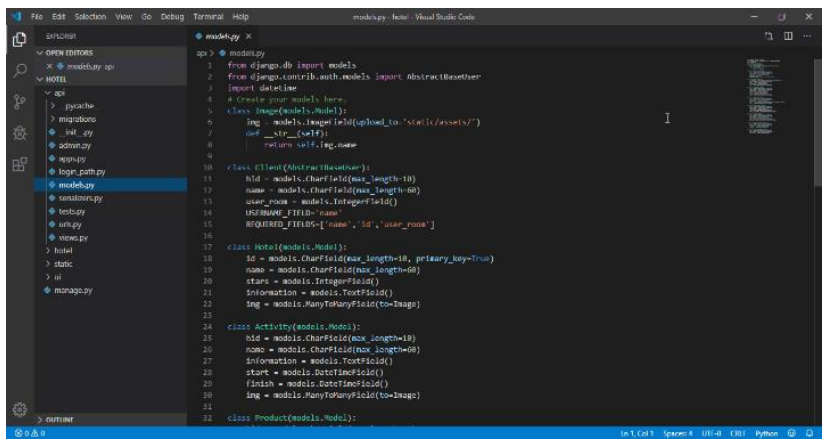

\section{Conclusiones}

- A pesar de no tratarse de una solución que ya se encuentre $100 \%$ operable si se logra dar una idea del proyecto y se argumenta todo el proceso de desarrollo llevado a cabo hasta la fecha.

- Se mencionan las herramientas utilizadas y se explica la problemática actual.

- Se deja en claro que se requiere la implementación de una solución informática para contrarrestar el problema y lograr un mayor desarrollo informático, así como mejorar los servicios y el gestionamiento en los hoteles de Cuba.

\section{Referencias bibliográficas}

Colombia.Travel. (2021). Uno de los mejores sitios web de turismo en el mundo - Viajar - Vida - ELTIEMPO.COM.”. https://www.eltiempo.com/vida/viajar/colombiatravel-uno-de-los-mejores-sitios-web-de-turismo-en-el-mundo-490396.

Geroldi, Michele. (2019). Páginas webs de turismo y viajes desarrolladas en WordPress. $\begin{array}{lllll}\text { Habitación } & 61 & \text { Blog de }\end{array}$ Sextaplantahttps://habitacion61.com/2019/04/09/paginas-webs-de-turismo-yviajes-desarrolladas-en-wordpress/. 
Xpertosolutions. (2021). La importancia de una página web en la empresa turística. https://www.xpertosolutions.com/x/noticia/item/la-importancia-de-una-pagina-weben-la-empresa-turistica.

Eumed. (2021). La web en destinos turísticos de cuba. https://www.eumed.net/rev/turydes/12/vbpg.html.

Mintur (2021) Sitio Oficial del Ministerio de Turismo de Cuba." Mintur. https://www.mintur.gob.cu/.

Django. (2021). The Web Framework for Perfectionists with Deadlines. https://www.djangoproject.com/. 
El artículo que se publica es de exclusiva responsabilidad de los autores y no necesariamente reflejan el pensamiento de la Revista Alfa Publicaciones.

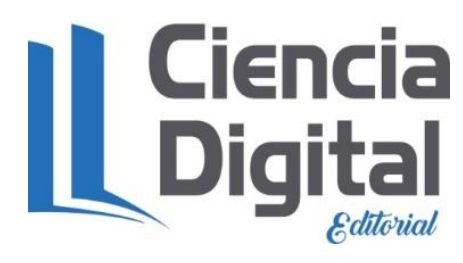

El artículo queda en propiedad de la revista y, por tanto, su publicación parcial y/o total en otro medio tiene que ser autorizado por el director de la Revista Alfa Publicaciones.
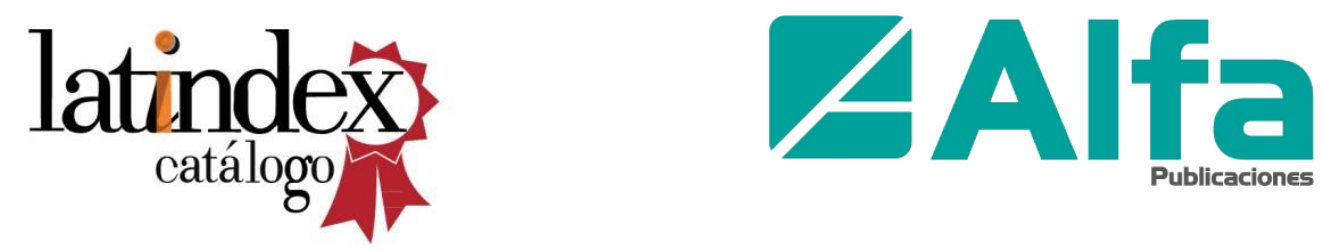

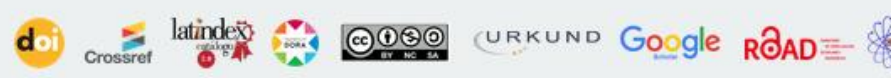
DLatinREV
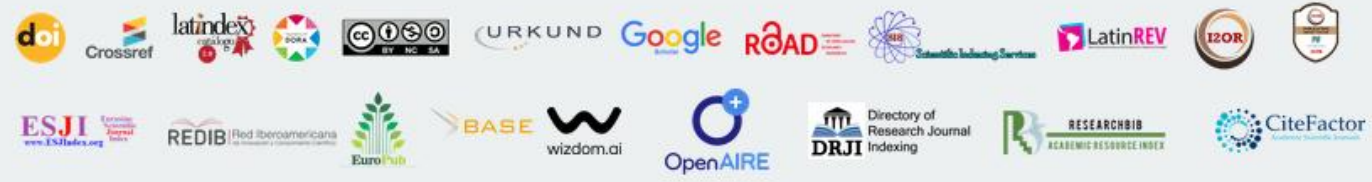